\title{
Protein profiling of paraquat-exposed rat lungs following treatment with Acai (Euterpe oleracea Mart.) berry extract
}

\author{
YONG-SIK KIM* ${ }^{*}$, HANA JUNG* , TAMANNA ZERIN and HO-YEON SONG \\ Department of Microbiology, College of Medicine, SoonChunHyang University, \\ Cheonan, Chung-Nam 330-090, Republic of Korea
}

Received July 12, 2012; Accepted November 6, 2012

DOI: $10.3892 / \mathrm{mmr} .2013 .1259$

\begin{abstract}
Paraquat (1,1'-dimethyl-4,4'-bipyridinium chloride, PQ) is a non-selective herbicide, and PQ poisoning by accidental or intentional ingestion is a cause of numerous fatalities around the world every year. Although a great deal of research has been conducted into the development of an acceptable treatment for PQ poisoning, no effective guidelines for patients have been developed thus far. Acai berry extract and juice have been highlighted in this regard, due to their observed antioxidant effects in various diseases. Furthermore, the acai berry has been used in dietary supplements, as it contains a variety of nutrients, including proteins, lipids, vitamins A, C and E and polyphenols. In this study, we conducted proteomic analysis of PQ-poisoned rat lungs to evaluate the changes in protein expression induced by PQ and to identify any protective effects of acai berry on the PQ poisoning. Our data revealed that the expression of the calcium signaling-related proteins calcium binding protein 1 (CaBP1), FK506 binding protein 4 (FKBP4), S100A6 and secreted protein acidic and rich in cysteine (Sparc, also known as osteonectin) were induced by PQ treatment and downregulated by acai berry treatment. However, the levels of protein kinase $\mathrm{C}$ substrate $80 \mathrm{~K}-\mathrm{H}$ were shown to be downregulated as the result of PQ treatment. Our results indicated that these proteins may function as biomarkers for acute poisoning by PQ exposure. Further studies may be necessary to understand their clinical relevance with regard to $\mathrm{PQ}$ poisoning.
\end{abstract}

\section{Introduction}

Paraquat dichloride (1,1'-dimethyl-4,4'-bipyridinium dichloride, $\mathrm{PQ})$, widely employed as an herbicide, is restricted in a

Correspondence to: Dr Ho-Yeon Song, Department of Microbiology, College of Medicine, SoonChunHyang University, 366-1 Ssangyong-dong, Cheonan, Chung-Nam 330-090, Republic of Korea

E-mail: songmic@sch.ac.kr

${ }^{*}$ Contributed equally

Key words: paraquat, acai berry, Euterpe oleracea Mart., proteomics, phytochemicals, Sparc, S100A6 number of countries but is frequently used to control weeds. It is fatal to humans when ingested, and is responsible for a high number of fatalities in developing countries (1). In humans, the $\mathrm{LD}_{50}$ of PQ is $3-5 \mathrm{mg} / \mathrm{kg}$ and its target organs are the kidney, liver, heart and lung. The principal molecular mechanism of PQ toxicity involves redox cycling and the generation of intracellular oxidative stress, and the lung is the primary target of PQ where it exerts a cytotoxic effect via the generation of reactive oxygen species (ROS) $(1,2)$. It has been demonstrated that the acute phase may cause mortality within a few days from pulmonary edema, cell infiltration and alveolar hemorrhage $(1,2)$. In the late phase of PQ poisoning, patients develop lung fibrosis followed by infiltrations of profibroblast cells with oxidative damage to lung tissues which contributes to extremely high morbidity and mortality. Despite intensive research into the pathobiological mechanisms of $\mathrm{PQ}$, no effective treatments for PQ poisoning have thus far been developed.

Phytochemicals have been highlighted as a possible therapeutic modality for a variety of diseases, including cancer and diabetes, due to their putative efficacies and safety. In particular, dietary phytochemicals, including resveratrol, (-)-epigallocatechin gallate (EGCG), [6]-gingerol, myricetin curcumin, quercetin and luteolin, have been recognized as antioxidants and directly regulate a variety of signal pathways in human diseases (3). The antioxidant function of the acai berry in a variety of human diseases has become the subject of some attention (4-8). The acai berry, the fruit of Euterpe oleraceae Martius, is a small round berry that grows in the Amazon region and is used medicinally as an antidiarrheal agent (8). Additionally, acai berry extract and juice have been used for foods and dietary supplements as they contain a variety of nutrients, including proteins, lipids, vitamins A, C and E, thiamine and flavan-3-ols, hydroxybenzoic acids, polyphenols and anthocyanins, predominantly cyanidin 3-O-rutinoside and cyanidin 3-O-glucuronide (9-11). Additionally, the acai berry has already been recognized as a possible pharmacological candidate in a variety of human diseases, including cancer, due to its strong ROS scavenging effect and anti-inflammatory effects induced via the regulation of cyclooxygenase (COX)-1 and -2 inhibitors $(4,11,12)$. In animal models, the acai berry has been shown to have a protective effect on cigarette-induced emphysema in mice (6). However, the acai berry has numerous potential effects in a variety of human diseases and its function in PQ poisoning has yet to be clearly elucidated. 
Proteomics is a technique that has been intensively employed in the past 10 years in a variety of life sciences in efforts to evaluate the mechanisms of the expression of genes and/or proteins that operate as regulators or modifiers during transcription, translation and post-translation $(13,14)$. The powerful and comprehensive information provided by proteomics research has been frequently applied to the discovery of new drugs, toxins and even treatments for human diseases such as cancer. This technique has emerged and expanded to other -omics, including epigenomics, metabolomics and genomics. In the toxicological sciences, proteomics is an appropriate technique for the discovery of target genes, biomarkers and therapeutic reagents. In the current study, among 40 spots, five proteins were identified the levels of which were altered $>2$-fold in the PQ-exposed rats treated with acai berry extract relative to the control group. Calcium binding protein 1 (CaBP1), FK506 binding protein 4 (FKBP4), secreted protein acidic and rich in cysteine (Sparc, also known as osteonectin) and S100A6 (also known as calcyclin) proteins were downregulated by acai berry, and Protein Kinase Substrate $80 \mathrm{H}-\mathrm{K}$ was upregulated by acai berry under PQ poisoning conditions. The data indicated that these proteins play pivotal roles in PQ-induced acute lung damage and may function as early biomarkers of PQ-induced injury. Furthermore, these data allowed us to speculate that the acai berry may contribute to the diminution of PQ-induced early lung cell damage via the regulation of the expression of these proteins and their functions. In order to gain insight into the mechanism of action of these proteins on PQ-poisoned acute lung damage and the clinical relevance of the acai berry in the context of PQ intoxication, further research will be required.

\section{Materials and methods}

Chemicals and reagents. MTT [3-(4,5-dimethylthiazol-2-yl)2,5-diphenyltetrazolium bromide] and PQ were purchased from Sigma-Aldrich (St. Louis, MO, USA).

Animals. Male CD(SD)IGS rats, 6-7 weeks old, were purchased from Orientbio (Seongnam, Korea) and allowed to acclimate to a standard rodent diet with free access to water for 7 days prior to the initiation of the experiment. The rats in the control group $(n=5)$ were treated with phosphate-buffered saline and sacrificed on the 7th day. The acai berry $(n=5)$ and PQ treatment $(n=5)$ groups were treated via intraperitoneal administration of $200 \mathrm{ng} / \mathrm{ml}$ PBS-diluted acai berry and $25 \mathrm{mg} / \mathrm{kg}$ PQ solution, respectively, and sacrificed on the 7th day. The $P Q$ with acai berry group $(n=5)$ rats were first treated via intraperitoneal injection of $25 \mathrm{mg} / \mathrm{kg}$ PQ solution followed by $200 \mathrm{ng} / \mathrm{ml}$ acai berry treatment $2 \mathrm{~h}$ and $6 \mathrm{~h}$ afterward. During the experiment, the rats were carefully monitored for signs of PQ poisoning. On the 7th day, the rats were sacrificed and the lungs were collected for proteomic analysis. Animal studies followed the guidelines of the Institutional Animal Care and Use Committee of Soonchunhyang University.

Cell culture. Human alveolar type II-like epithelial A549 cells (ATCC\# CCL-185) were purchased from the American Type Culture Collection (Manassas, VA, USA) and the cells were maintained in standard DMEM/F-12 Ham supplemented with $10 \%(\mathrm{v} / \mathrm{v})$ heat-inactivated FBS and $1 \%(\mathrm{v} / \mathrm{v})$ antibiotics (100 U/ml penicillin, $100 \mu \mathrm{g} / \mathrm{ml}$ streptomycin and $0.25 \mu \mathrm{g} / \mathrm{ml}$ amphotericin B; Invitrogen, Carlsbad, CA, USA) at $37^{\circ} \mathrm{C}$ in an incubator with a humidified atmosphere of $5 \% \mathrm{CO}_{2}$.

MTT assay. The MTT assay was carried out at various time-points, including 12, 24, 48 and $72 \mathrm{~h}$ after PQ treatment coupled with acai berry treatment. In brief, $80 \%$ confluent A549 lung adenocarcinoma cells were seeded on 96-well plates prior to the beginning of the experiment. The cells were treated with PQ and acai berry time- and dose-dependently and then were stained for $2 \mathrm{~h}$ with MTT $(2 \mathrm{mg} / \mathrm{ml})$. The medium was removed and the formazan crystals produced were dissolved via the addition of $200 \mu \mathrm{l}$ dimethyl sulfoxide. The absorbance at $540 \mathrm{~nm}$ was then measured using a SpectraMax 250 microplate reader (Molecular Probes, Eugene, OR, USA).

\section{Proteomics}

Sample preparation. Tissues were homogenized in sample lysis buffer and the proteins were extracted. Insoluble material was discarded via centrifugation at 15,000 $\mathrm{x}$ g for $1 \mathrm{~h}$ and the concentration of proteins was measured via Bradford assay (Bio-Rad, Hercules, CA, USA).

$2 D$ PAGE. Isoelectric focusing (IEF) using $200 \mu \mathrm{g}$ samples was conducted at $20^{\circ} \mathrm{C}$ using a Multiphor II electrophoresis unit in accordance with the manufacturer's instructions (Amersham Biosciences, Pittsburgh, PA, USA). Prior to running the second dimension, the strips were incubated for $10 \mathrm{~min}$ with equilibration buffer $(50 \mathrm{mM}$ Tris-Cl, $\mathrm{pH} 6.8$, containing $6 \mathrm{M}$ urea, $2 \%$ SDS and 30\% glycerol), first with $1 \%$ DTT and second with $2.5 \%$ iodoacetamide. Equilibrated strips were inserted onto SDS-PAGE gels (20x24 cm, 10-16\%) and 2D gels were run. When the runs were completed, the 2D gels were subjected to silver staining via a modification of the method devised by Oakley et al (15).

Image analysis. Digitalized images were quantitatively analyzed using the PDQuest (version 7.0, Bio-Rad) software in accordance with the manufacturer's instructions. Each spot was normalized by total valid spot intensity and the spots whose expression levels had altered $>2$-fold were selected.

In-gel digestion. Protein spots were enzymatically digested in-gel in a manner similar to that described previously (16) and were modified by porcine trypsin (Promega, Madison, WI, USA). The gel pieces were washed with 50\% acetonitrile and subsequently dehydrated. The spots were then dried to remove the solvent, rehydrated with trypsin (8-10 $\mathrm{ng} / \mu \mathrm{l})$ solution in $50 \mathrm{mM}$ ammonium bicarbonate $(\mathrm{pH} 8.7)$ and then incubated for $8-10 \mathrm{~h}$ at $37^{\circ} \mathrm{C}$.

Identification of proteins by MALDI-TOF/TOF. Samples were analyzed using an Applied Biosystems 4700 proteomics analyzer with TOF/TOFTM ion optics. MS and MS/MS data were acquired with a Nd:YAG laser with a repetition rate of $200 \mathrm{~Hz}$ and up to 4000 shots were accumulated for each spectrum. MS and MS/MS data were acquired using the instrument default calibration, without applying internal or external calibration. Sequence tag searches were conducted using the MASCOT program run by the National Resource for the Mass Spectrometric Analysis of Biological Macromolecules and Matrixscience Company (http://www.matrixscience.com/). 


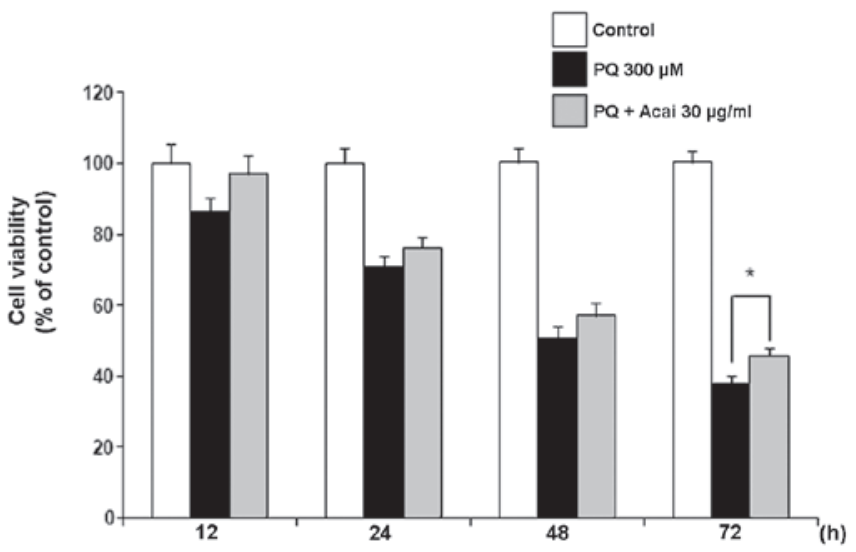

Figure 1. Lung carcinoma A549 cell viability following acai berry treatment. Cells were seeded on 96-well plates prior to the performance of the 3-(4,5-dimethylthiazol-2-yl)-2,5-diphenyltetrazolium bromide (MTT) assay. After $12 \mathrm{~h}, 300 \mu \mathrm{M}$ paraquat (PQ) was administered, followed by immediate treatment with acai berry at the indicated doses. MTT was administered at $12,24,48$ and $72 \mathrm{~h}$ and measured. The blank bars indicate control cells, black bars indicate PQ-treated cells and gray bars indicate cells treated with PQ and $30 \mu \mathrm{g} / \mathrm{ml}$ acai berry. ${ }^{*} \mathrm{P}<0.05$.

Quantitative RT-PCR. Total RNAs were extracted from lung tissues using RNA isolation kits (Qiagen, Hilden, Germany). cDNAs were generated using the first-strand cDNA synthesis kit (Intron, Seongnam, Korea) and qRT-PCR assays were conducted using a Bio-Rad real-Time PCR kit with a rat Sparc-specific primer set comprising: forward primer, 5'-GTGTGCAGCAATGACAACAAG-3'; and reverse primer; 5'-TCAGCTCAGAATCCAGGCAG-3').

Statistical analyses. The results were shown as the means \pm standard deviation (SD) from triplicate samples of three independent experiments. Statistical significance was analyzed using a paired Student's t-test with $\mathrm{P}<0.05$ considered as significant.

\section{Results}

Acai berry has a protective effect against $P Q$-induced A549 cytotoxicity. In order to investigate the protective effects of the acai berry, we initially conducted an MTT cytotoxicity assay of PQ-treated lung adenocarcinoma A549 cells (Fig. 1). Prior to performing these experiments, we tested the toxicity of the acai berry to the A549 cells by MTT assay. Concentrations of the acai berry $\leq 50 \mu \mathrm{g} / \mathrm{ml}$ evidenced no cytotoxicity (data not shown). Acai berry and PQ co-treatment was also evaluated. Fig. 1 shows that the viability of the cells was reduced markedly in the PQ-treated group. The cell viability began to reduce at $12 \mathrm{~h}$ and reached almost $40 \%$ at $72 \mathrm{~h}$, whereas the cell viability of the control cells did not change. The viability of the A549 cells treated with PQ and $30 \mu \mathrm{g} / \mathrm{ml}$ acai berry extract was unchanged at $12 \mathrm{~h}$ compared with that of the control cells, but subsequently decreased gradually until $72 \mathrm{~h}$. However, the cytotoxicity of PQ was markedly diminished by co-treatment with acai berry relative to that of the group treated with PQ alone at $72 \mathrm{~h}$. The data indicated that acai berry exerts a protective effect against PQ-induced cell cytotoxicity.

In order to assess the clinical relevance of the results, we designed an animal model system. We used CD(SD)IGS rats

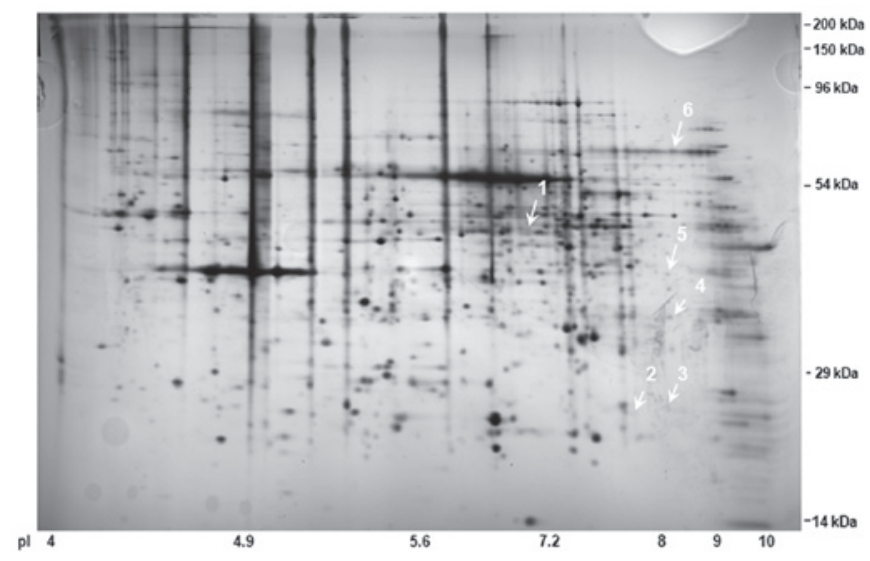

Figure 2. Analysis of a representative 2DE gel of rat lung proteins treated with paraquat (PQ) and acai berry. Differentially expressed proteins were visualized via a modified silver staining method. Arrows indicate the proteins that evidenced substantially altered expression ( $>2$-fold) relative to the control and treatment groups.

assigned randomly to the following groups prior to administration: acai berry (group A, n=5), PQ (group PQ, n=5), PQ with acai berry (group PA, $n=5$ ) and control (group $C, n=5$ ). After acclimation, the rats of group PA were treated via the intraperitoneal administration of $200 \mathrm{ng} / \mathrm{ml}$ PBS-diluted acai berry at 2 and $6 \mathrm{~h}$ later, $25 \mathrm{mg} / \mathrm{kg}$ PQ was administered. Following PQ administration, the rats were carefully monitored for any signs of PQ poisoning. On the 7th day, the rats were euthanized and their lungs were collected. In order to confirm PQ poisoning, we conducted micro-CT analysis of the rat lungs. Notably, we observed no significant differences in the lung tissues among the groups (data not shown). The data indicated that the low dose of PQ treatment may not induce severe or acute lung phenotypes. However, we subsequently conducted proteomic analysis of the rat lung tissues, since global gene expression may be altered during chemical treatments before the tissues demonstrate any severe pathological features.

Proteomic analysis. In order to assess whether gene expression was altered during the PQ-induced acute lung injury, we conducted duplicate conventional proteomic analyses using $200 \mu \mathrm{g}$ protein samples from the four groups. In Fig. 2, a combined 2D-SDS PAGE image is presented with $>800$ spots. By comparing the duplicated 2D-SDS-PAGE gels, we analyzed and identified 6 differentially expressed protein spots among the 40 spots, the expression levels of which were substantially altered $>2$-fold relative to basal expression levels. The substantially altered protein expression spots are shown in Fig. 3A and the relative volumes of the spots are shown in Fig. 3B. These spots consisted of one upregulated and five downregulated protein spots for the PA group. To characterize the spots, we utilized MS/MS-TOF and analyzed the spots via the MASCOT analysis program. The results of Mascot analysis revealed that the upregulated spot for the PA group was protein kinase $\mathrm{C}$ substrate $80 \mathrm{~K}-\mathrm{H}$ protein with a Mowse score of $53(\mathrm{P}<0.05$; Table I). Spot 2 was matched with CaBP1 with a Mowse score of $44(\mathrm{P}<0.05)$ and the peptides covered $3 \%$ of the entire CaBP1 protein. Spot 4 was identified as FKBP4 with a score of $34(\mathrm{P}<0.05)$ and a peptide 
Table I. A list of characterized and altered proteins from PQ-induced lung injury in rats following acai berry treatment

\begin{tabular}{lllccc}
\hline SPOT & Annotation & \multicolumn{1}{c}{ Identified protein } & $\begin{array}{c}\text { Mowse } \\
\text { score }\end{array}$ & Coverage (\%) & $\begin{array}{c}\text { Change in } \\
\text { group PA }\end{array}$ \\
\hline 1 & gil149020437 & Protein kinase C substrate 80K-H & 53 & 2 & Up \\
2 & gil488838 & CaBP1 & 44 & 3 & Down \\
3 & & No significant hits & - & - & Down \\
4 & gil22324680 & FK506 binding protein 4 & 34 & 3 & Down \\
5 & gil600381 & Sparc (osteonectin) & 416 & 14 & Down \\
6 & gil16758986 & S100-A6 & 30 & Down \\
\hline
\end{tabular}

PQ, paraquat; CaBP1, calcium binding protein 1. Group PA, rats treated with paraquat and acai berry.
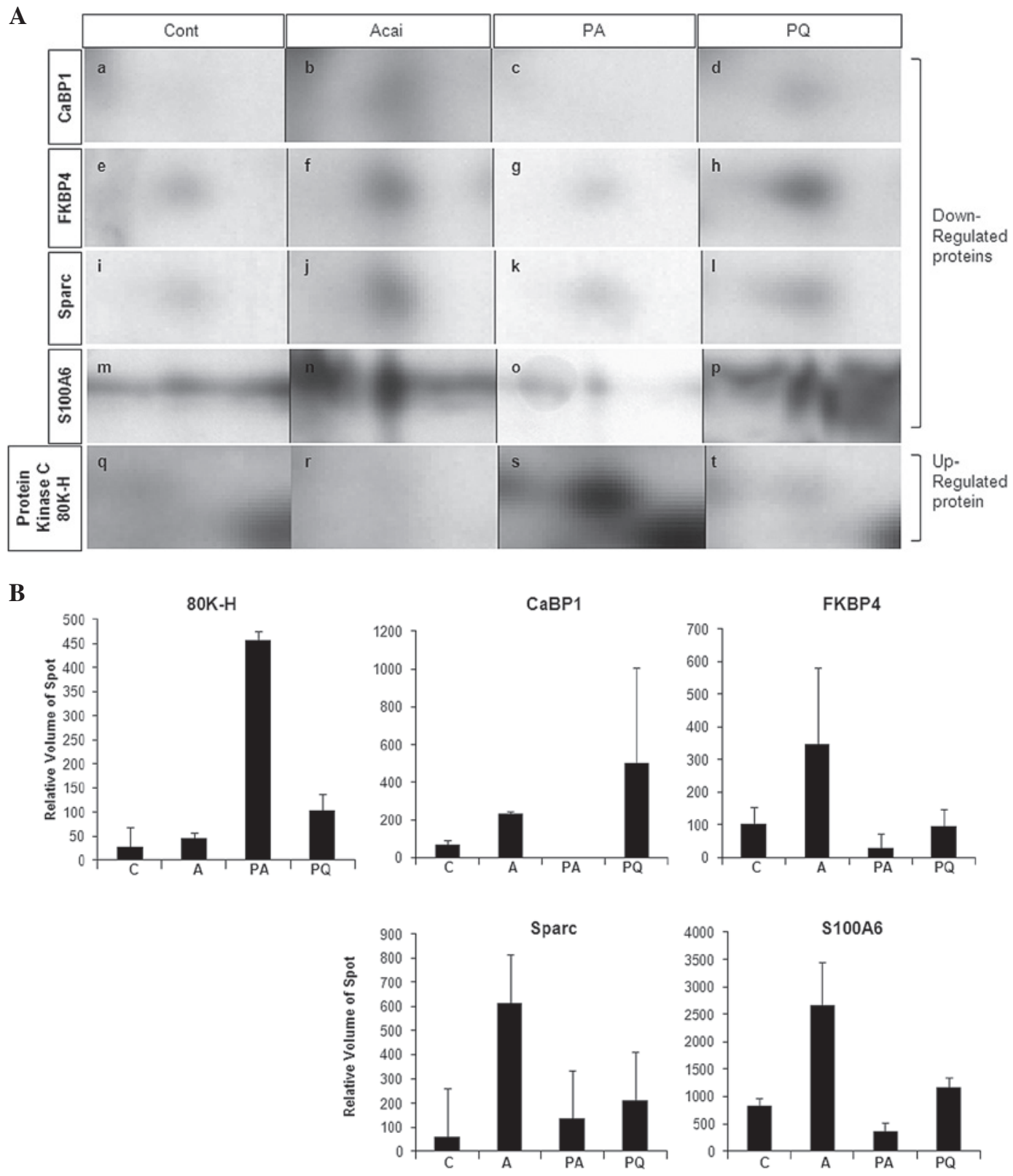

Figure 3. (A) Determination of upregulated and downregulated proteins in rat lungs treated with paraquat (PQ), acai berry (A) or PQ and acai berry (PA). Downregulated proteins in the lung samples of the PA group rats are shown in (a-p). These downregulated proteins comprise (a-d) calcium binding protein 1 (CaBP1); (e-h) FK506 binding protein 4 (FKBP4); (i-l) Sparc and (m-p) S100A6. (q-t) The upregulated protein in group PA is protein kinase C substrate 80K-H. (B) The quantitative alteration of selected protein expression levels in $\mathrm{PQ}$-treated rat lung protein samples. Relative volumes of spots for protein kinase $\mathrm{C}$ substrate $80 \mathrm{~K}-\mathrm{H}, \mathrm{CaBP} 1$, FKBP4, Sparc and S100A6. Protein kinase C substrate 80K-H was upregulated in the PA samples but not in the control (C) and acai berry only treatment groups. CaBP1, FKBP4, Sparc and S100A6 protein spots were upregulated in the PQ- and acai berry-treated lung protein samples, respectively. 


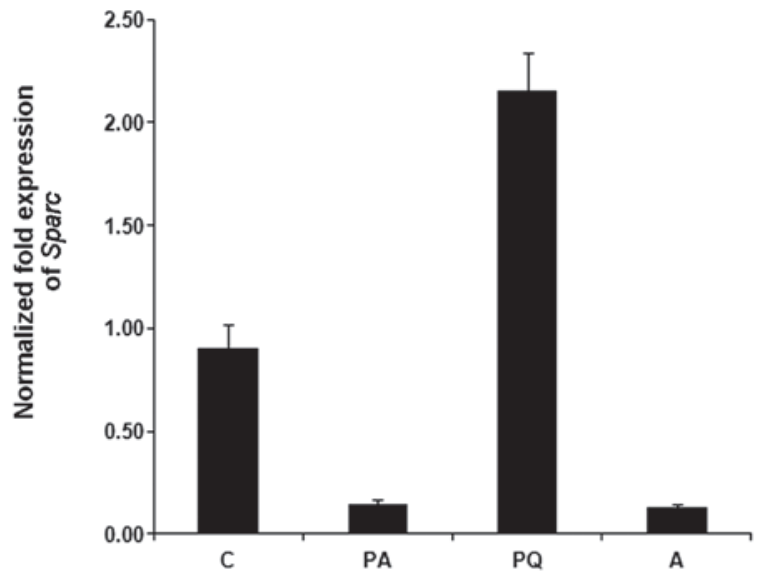

Figure 4. Expression of Sparc mRNA by paraquat (PQ)-treated rat lungs RNA was purified from the tissues and quantitative RT-PCR was conducted using a Sparc primer set as described in Materials and methods. C, A, PA and $\mathrm{P}$ correspond to the control and acai berry-, PQ with acai berry- and PQ-treated groups, respectively.

coverage of $3 \%$. Notably, spot 5 matched highly with Sparc with a Mowse score of $116(\mathrm{P}<0.05)$ and a peptide coverage of $14 \%$ from the 301 amino acid polypeptide. S100A6 (spot 6) was characterized by a score of $47(\mathrm{P}<0.05)$ and $30 \%$ peptide coverage. However, spot 3 was not characterized by Mascot analysis, owing to a non-significant match to the proteins in the protein database.

In order to confirm the alteration of protein expression in vivo, we conducted quantitative RT-PCR using a Sparc-specific primer set (Fig. 4). This revealed that Sparc mRNA was induced in the PQ group but not in the acai berry-treated group. However, Sparc gene expression was altered slightly in the PA rat group.

\section{Discussion}

PQ is broadly employed as an herbicide for weed control all over the world, particularly in third world countries, and remains one of the principal causes of fetal mortality in humans as the result of accidental or intentional ingestion (8). Acute PQ poisoning in animal and human models leads to mortality within a few days due to severe pulmonary fibrosis, but the early molecular mechanisms of PQ-induced lung fibrosis remain incompletely understood. Furthermore, studies of the molecular mechanisms relevant to PQ-induced lung fibrosis using in vitro and in vivo animal models have yet to generate clear results. Thus far, intensive research has been conducted to develop treatments for PQ poisoning, but no effective treatments have been developed.

Proteomics is a powerful tool for the analysis of global gene expression and the display of differential protein expression governed by functional genes in comparison among groups, as well as heterogeneous diseases such as diabetes and cancer (14). Proteomics has been highlighted and expanded into a variety of research areas for clinical application. In this regard, proteomics is an extremely useful tool for the discovery of biomarkers, pharmacological targets and drugs. However, few studies have been conducted in which the conventional proteomics analysis technique has been employed in the context of PQ-induced poisoning. Hence, we utilized proteomics technology to investigate proteins that are differentially expressed during treatment with PQ and/or acai berry extract.

In this study, combined 2DE gels revealed $\sim 800$ spots that were be identified via MALDI-TOF/TOF analysis. We selected 40 spots that were found have $>2$-fold altered expression and 6 spots were ultimately selected for further investigation. The most highly matched spot was Sparc; the spot covered $14 \%$ of the peptide portion on the entire Sparc protein with a Mowse score of 116. Sparc is a secreted glycoprotein with multiple functions in tissue remodeling, wound healing, cell proliferation and a variety of diseases, including cancer (17). Although the biological functions of Sparc remain controversial, its functions have been demonstrated in the context of idiopathic pulmonary fibrosis via the suppression of apoptosis through the activation of $\beta$-catenin (18). The results of in vivo and in vitro studies using siRNA and null mice have demonstrated that Sparc gene silencing may significantly reduce collagen expression $(19,20)$. A growing body of data has accumulated to suggest that Sparc is a biomarker for fibrosis. Our findings suggest that Sparc expression was induced by PQ treatment but reduced by acai berry treatment and indicate that Sparc may be a useful biomarker of PQ-induced poisoning.

S100A6 consists of 90 amino acids and is a member of the S100 domain family within the EF-hand calcium binding protein superfamily, which is associated with a variety of cellular and extracellular processes (21). Researchers have demonstrated that S100A6 performs crucial functions in pulmonary fibroblast proliferation and responds to stress conditions, including mechanical tension in pulmonary IMR-90 cells and oxidation by exobiotics (22-24). It has been demonstrated that the activation of S100A6 promotes apoptosis in cells under oxidative stress and also induces the ROS-dependent activation of JNK and caspases -3 and -7 in neuroblastoma cells (25).

Proteomics and MS/MS analysis results indicated that S100A6 was downregulated in the PQ plus acai berry treatment group. In Fig. 3B, the S100A6 expression induced by PQ treatment is revealed to be repressed $\sim 5$-fold by acai berry treatment. The data indicate that acai berry may exploit a mechanism that represses PQ-induced apoptosis and ultimately exerts a protective effect against PQ poisoning. However, in order to define the mechanism of S100A6 regulation utilized by the acai berry, further study may be necessary.

$\mathrm{CaBP} 1$ is a calcium -binding protein and carries out a modulatory function in the gating of voltage-gated calcium channels $(26,27)$. However, the function of CaBP1 in other physiological contexts and its implication with regard to human diseases has yet to be thoroughly evaluated. Our proteomics results showed that $\mathrm{PQ}$-induced $\mathrm{CaBP} 1$ expression was downregulated by acai berry treatment. These data lead us to speculate that the acai berry may deliver and trigger signaling via a calcium-dependent antioxidant pathway. However, further studies of the functions of $\mathrm{CaBP} 1$ in the PQ-induced ROS signal pathway are required.

FKBP4 (FKBP52) is a member of the immunophilin proteins and functions as a co-chaperone with heat-shock protein 90 (HSP90) (28). The results of a recent study has 
demonstrated that FKBP52 regulates oxidative stress via the unique antioxidant, peroxiredoxin-6 (PRDX6), which functions independently of other peroxiredoxins and antioxidant proteins (29). However, FKBP4 expression was shown to be downregulated by acai berry treatment in this study, thus suggesting the existence of an alternative antioxidant pathway exploited by the acai berry under PQ exposure conditions.

The protein kinase $\mathrm{C}$ substrate $80 \mathrm{~K}-\mathrm{H}(80 \mathrm{~K}-\mathrm{H})$ was the only upregulated protein spot identified in the PA group in this study. The protein kinase $\mathrm{C}$ substrate $80 \mathrm{~K}-\mathrm{H}(80 \mathrm{~K}-\mathrm{H})$ forms a complex with the epithelial $\mathrm{Ca}^{2+}$ channel transient receptor potential cation channel V5 (TRPV5) and is able to regulate the activity of the channel (30).

Barnard et al demonstrated that the mechanism underlying the protective effect of the acai berry against PQ involves a calcium-dependent reaction (31). Notably, in this study, the calcium regulation-related genes, $\mathrm{S} 100 \mathrm{~A} 6, \mathrm{CaBP} 1$ and protein kinase $\mathrm{C}$ substrate $80 \mathrm{~K}-\mathrm{H}$, were substantially altered by either PQ or the acai berry. The data indicate that the acai berry may employ calcium signaling for its protective effects against PQ-induced ROS signaling.

In summary, the proteomic data for the herbicide PQ and phytochemical acai berry extract have allowed us to propose several target biomarkers for acute PQ poisoning. Furthermore, our data indicate the possibility that the acai berry contains a therapeutic phytochemical reagent that may help to alleviate or protect against PQ intoxication.

\section{Acknowledgements}

The authors would like to express their gratitude to Dr Gil, Hyo-Wook (SoonChunHyang University Hospital, Cheonan, Korea) for his valuable comments.

\section{References}

1. Dinis-Oliveira RJ, Duarte JA, Sánchez-Navarro A, Remião F, Bastos ML and Carvalho F: Paraquat poisonings: mechanisms of lung toxicity, clinical features, and treatment. Crit Rev Toxicol 38: 13-71, 2008.

2. Smith LL: Mechanism of paraquat toxicity in lung and its relevance to treatment. Hum Toxicol 6: 31-36, 1987.

3. Lee KW, Bode AM and Dong Z: Molecular targets of phytochemicals for cancer prevention. Nat Rev Cancer 11: 211-218, 2011.

4. Xie C, Kang J, Burris R, et al: Acai juice attenuates atherosclerosis in ApoE deficient mice through antioxidant and anti-inflammatory activities. Atherosclerosis 216: 327-333, 2011.

5. Udani JK, Singh BB, Singh VJ and Barrett ML: Effects of Acai (Euterpe oleracea Mart.) berry preparation on metabolic parameters in a healthy overweight population: A pilot study. Nutr J 10: 45, 2011.

6. de Moura RS, Pires KM, Santos Ferreira T, et al: Addition of açaí (Euterpe oleracea) to cigarettes has a protective effect against emphysema in mice. Food Chem Toxicol 49: 855-863, 2011.

7. Schauss AG, Clewell A, Balogh L, et al: Safety evaluation of an açai-fortified fruit and berry functional juice beverage (MonaVie Active $\left({ }^{\circledR}\right)$ ). Toxicology 278: 46-54, 2010 .

8. Schauss AG, Wu X, Prior RL, et al: Phytochemical and nutrient composition of the freeze-dried amazonian palm berry, Euterpe oleraceae mart. (acai). J Agric Food Chem 54: 8598-8603, 2006.
9. Del Pozo-Insfran D, Brenes CH and Talcott ST: Phytochemical composition and pigment stability of Açai (Euterpe oleracea Mart.). J Agric Food Chem 52: 1539-1545, 2004.

10. Lichtenthäler R, Rodrigues RB, Maia JG, Papagiannopoulos M, Fabricius $\mathrm{H}$ and Marx F: Total oxidant scavenging capacities of Euterpe oleracea Mart. (Açaí) fruits. Int J Food Sci Nutr 56: 53-64, 2005.

11. Rodrigues RB, Lichtenthäler R, Zimmermann BF, et al: Total oxidant scavenging capacity of Euterpe oleracea Mart. (açaí) seeds and identification of their polyphenolic compounds. J Agric Food Chem 54: 4162-4167, 2006.

12. Sun X, Seeberger J, Alberico T, et al: Açai palm fruit (Euterpe oleracea Mart.) pulp improves survival of flies on a high fat diet. Exp Gerontol 45: 243-251, 2010.

13. Banks RE, Dunn MJ, Hochstrasser DF, et al: Proteomics: new perspectives, new biomedical opportunities. Lancet 356: 1749-1756, 2000

14. Plymoth A and Hainaut P: Proteomics beyond proteomics: toward clinical applications. Curr Opin Oncol 23: 77-82, 2011.

15. Oakley BR, Kirsch DR and Morris NR: A simplified ultrasensitive silver stain for detecting proteins in polyacrylamide gels. Anal Biochem 105: 361-363, 1980.

16. Shevchenko A, Wilm M, Vorm O and Mann M: Mass spectrometric sequencing of proteins silver-stained polyacrylamide gels. Anal Chem 68: 850-858, 1996.

17. Clark CJ and Sage EH: A prototypic matricellular protein in the tumor microenvironment - where there's SPARC, there's fire. J Cell Biochem 104: 721-732, 2008.

18. Chang W, Wei K, Jacobs SS, Upadhyay D, Weill D and Rosen GD: SPARC suppresses apoptosis of idiopathic pulmonary fibrosis fibroblasts through constitutive activation of beta-catenin. J Biol Chem 285: 8196-8206, 2010.

19. Wang JC, Lai S, Guo X, et al: Attenuation of fibrosis in vitro and in vivo with SPARC siRNA. Arthritis Res Ther 12: R60, 2010.

20. Savani RC, Zhou Z, Arguiri E, et al: Bleomycin-induced pulmonary injury in mice deficient in SPARC. Am J Physiol Lung Cell Mol Physiol 279: L743-L750, 2000.

21. Leśniak W, Słomnicki ŁP and Filipek A: S100A6 - new facts and features. Biochem Biophys Res Commun 390: 1087-1092, 2009.

22. Zhang SP, Wu YW, Wu ZZ, Liu HY, Nie JH and Tong J: Up-regulation of RAGE and S100A6 in rats exposed to cigarette smoke. Environ Toxicol Pharmacol 28: 259-264, 2009.

23. Leśniak W, Szczepańska A and Kuźnicki J: Calcyclin (S100A6) expression is stimulated by agents evoking oxidative stress via the antioxidant response element. Biochim Biophys Acta 1744: 29-37, 2005.

24. Breen EC and Tang K: Calcyclin (S100A6) regulates pulmonary fibroblast proliferation, morphology, and cytoskeletal organization in vitro. J Cell Biochem 88: 848-854, 2003.

25. Słomnicki ŁP, Nawrot B and Leśniak W: S100A6 binds p53 and affects its activity. Int J Biochem Cell Biol 41: 784-790, 2009.

26. Haeseleer F, Sokal I, Verlinde CL, et al: Five members of a novel $\mathrm{Ca}\left({ }^{2+}\right)$-binding protein (CABP) subfamily with similarity to calmodulin. J Biol Chem 275: 1247-1260, 2000.

27. Oz S, Tsemakhovich V, Christel CJ, Lee A and Dascal N: $\mathrm{CaBP} 1$ regulates voltage-dependent inactivation and activation of $\mathrm{Ca}(\mathrm{V}) 1.2$ (L-type) calcium channels. J Biol Chem 286: 13945-13953, 2011.

28. Davies TH and Sanchez ER: Fkbp52. Int J Biochem Cell Biol 37: 42-47, 2005.

29. Hirota Y, Acar N, Tranguch S, et al: Uterine FK506-binding protein 52 (FKBP52)-peroxiredoxin-6 (PRDX6) signaling protects pregnancy from overt oxidative stress. Proc Natl Acad Sci USA 107: 15577-15582, 2010

30. Gkika D, Mahieu F, Nilius B, Hoenderop JG and Bindels RJ: $80 \mathrm{~K}-\mathrm{H}$ as a new $\mathrm{Ca}^{2+}$ sensor regulating the activity of the epithelial $\mathrm{Ca}^{2+}$ channel transient receptor potential cation channel V5 (TRPV5). J Biol Chem 279: 26351-26357, 2004.

31. Barnard JW, Womack WA, Smith SM, Engerson TD and Taylor AE: Lung protection against paraquat is calcium dependent. J Appl Physiol 72: 498-504, 1992. 